\title{
Novel biomarkers for prediction of acute kidney injury in acute heart failure
}

\author{
Dankova $\mathrm{M}^{1}$, Minarikova $\mathrm{Z}^{2}$, Danko $\mathrm{J}^{3}$, Gergel $\mathrm{J}^{4}$, Pontuch $\mathrm{P}^{2}$, Goncalvesova $\mathrm{E}^{1}$ \\ Department of Cardiology, School of Medicine, Comenius University and National Cardiovascular Institute, \\ Bratislava, Slovakia. marcela.dankova@nusch.sk
}

\section{ABSTRACT}

OBJECTIVES: Acute kidney injury (AKI) is a frequent event in patients with an acute heart failure (AHF) and is associated with a poor short and long-term outcome. The aim of this study was to describe diagnostic yield of selected novel biomarkers in prediction of AKI in patients admitted for AHF.

METHODS: We performed a prospective cohort study of 72 consecutive patients (46/26 M/F) aged $69 \pm 10,3$ years admitted for AHF. Renal damage was defined according to KDIGO guidelines. Patients were divided into the two groups: $A K I-$ (without renal injury, $n=52$ ) and $A K I+$ (with renal injury, $n=20$ ). Urine samples for AKI biomarkers measurements (NGAL, TIMP2, IGFBP7) were collected at the admission. The ROC and linear logistic regression of new biomarkers and selected clinical variables was performed for the evaluation of the AKI prediction.

RESULTS: The patients with AKI+ were older (median age: 75 vs 64 years, $p=0.01$ ), had lower BMI (median: 28 vs $29.5 \mathrm{~kg} / \mathrm{m}^{2}, p=0.04$ ), were with a higher proportion of patients with HF with a reduced ejection fraction ( $55 \%$ vs $23.1 \%, p=0.01$ ) and a higher level of serum NTproBNP. Urinary NGAL at admission was significantly higher in the AKI+ compared to the AKI - group (152 vs $19.5 \mathrm{ng} / \mathrm{mL}, p<0.0001$ ); also median of U-TIMP-2 and U-IGFBP-7 in the AKI+ patients was significantly higher: 194.1 versus $42.5 \mathrm{ng} / \mathrm{mL}(p<0.0001)$ and 379 versus $92.4 \mathrm{pg} / \mathrm{mL}$ ( $p<0.0001$ ) resp. Age, u-NGAL, u-TIMP2, u-IGFBP7, s-haemoglobin, NTproBNP and LVEF were associated with the development of AKI. Urine concentration of IGFBP-7 was measured, which is the best marker for the prediction of AKI (AUC 0.94). CONCLUSION: Urine concentrations of NGAL, TIMP2, IGFBP7 at the time of admission for AHF predicted a development of AKI. Age, NTproBNP, LVEF and s-haemoglobin were also associated with AKI in AHF patients (Tab. 3, Fig. 3, Ref. 22). Text in PDF www.elis.sk. KEY WORDS: biomarkers, cardiorenal syndrome, acute heart failure.

\section{Introduction}

Acute heart failure (AHF) represent a frequent cause of hospitalisations and it is associated with a high risk of acute kidney injury (AKI). Acute kidney injury and/ or kidney failure is a frequent clinical entity in AHF patients, which prolongs hospitalisation and increases mortality in this high- risk group (1). The incidence of AKI in patients hospitalised due to an acute decompensated heart failure is reported by some authors up to $40 \%$ (2). The causes of AKI in AHF are multifactorial and complex, mechanism varies from patient to patient. Early detection of patients with acute

${ }^{1}$ Department of Cardiology, School of Medicine, Comenius University and National Cardiovascular Institute, Bratislava, Slovakia, ${ }^{2}$ Department of Internal Medicine IV, School of Medicine, Comenius University and University Hospital, Bratislava, Slovakia, ${ }^{3}$ Slovak University of Technology, Bratislava, Slovakia, and ${ }^{4}$ Medirex, OJSC, Bratislava, Slovakia

Address for correspondence: M. Danková, MD, PhD, Department of Cardiology, School of Medicine, Comenius University and National Cardiovascular Institute, Pod Krásnou hôrkou 1, SK-833 48 Bratislava, Slovakia. Phone: +421259320268 , Fax: +421254788737

Acknowledgement: Project was supported by Slovak Society of Cardiology research grant 2015-2018. cardiorenal syndrome is crucial for their prognosis (3). Serum creatinine as an early marker of acute kidney injury is insufficient, because of its delayed serum increase after the initiating insult (4). That was the primary impetus for the discovery of novel biomarkers. The goal of most AKI biomarker research has been discovery of a ,troponin of the kidney", a sensitive and specific early marker of renal injury. Despite the precision of the new biomarkers, the expectation, that they become kidney troponin will already fall on the principal differences in the pathophysiology of AKI and myocardial infarction. Additionally, biomarkers are qualitatively different molecules such as troponins. The induction mechanism of AKI is not one disease entity, but it is a clinical syndrome with a myriad of multifactorial renal insults. Therefore, it is not easy to find one marker with the diagnostic accuracy of troponin. After more than a decade of intensive research effort, several biomarkers as neutrophil gelatinase associated lipocalin (NGAL), kidney injury molecule 1 (KIM-1), interleukin 18 (IL 18) have been identified. Unfortunately, the clinical use of novel AKI biomarkers remains unclear (5). Tissue metalloproteinase 2 inhibitor (TIMP-2) and insulin like growth factor binding protein 7 (IGFBP-7) are markers of cycle cell arrest in the G1 phase and are also a relatively new group of biomarkers in the early detection of AKI. TIMP-2 plays 
an important role in the pathophysiology of ischemic-reperfusion injury and IGFBP-7 is a secretory protein that regulates the bioavalability of insulin-like growth factors $(6,7)$. The aim of the study was to describe the effectivity of selected novel biomarkers in prediction of AKI in patients admitted for acute HF.

\section{Patients and methods}

In the prospective cohort study, 72 patients admitted due to acute heart failure were enrolled. An acute decompensated heart failure was defined as an acute decompensation of known heart failure or newly diagnosed acute heart failure. Patients were included with typical clinical symptoms (dyspnoea, orthopnoea, paroxysmal night dyspnoea), physical signs of fluid retention and $\mathrm{X}$-ray correlate after obtaining the written informed consent. Patients with a history of chronic kidney disease in KDIGO 4 and 5 stages, patients with an acute infection of urogenital tract, patients with a history of kidney or bladder cancer and patients with ongoing sepsis were excluded. Urine samples for AKI biomarkers measurements (NGAL, TIMP2, IGFBP7) were collected at the admission. Urine samples were centrifuged and supernatants were frozen by standard methods within 0,5 hour of collection and stored at $-70^{\circ} \mathrm{C}$. The samples were assayed by enzyme-linked immuno sorbent assay (NGAL Rapid ELISA Kit, IGFBP-7 ELISA Kit, Human TIMP-2 ELISA Kit). Biochemical analysis of the blood samples included serum creatinine, N-terminal pro brain natriuretic peptide, haemoglobin at the admission. Serum creatinine evaluation was also performed after 24, 48 and 72 hours later. Patients were divided into the two groups (with acute kidney injury AKI+ and without kidney injury AKI -), according to the KDIGO guidelines based on the creatinine levels and daily diuresis (4).

Echocardiographic examination was carried out within first 24 hours of the hospitalization. Statistical analysis was done in the JMP Pro 13. Difference in mean between two quantitative variables was evaluated using unpaired Student's t-test for normally distributed data and the non-parametric Mann-Whitney test for abnormally distributed data. The ROC analysis was calculated to determine the discriminatory ability of biomarkers. Linear logistic regression was performed in the AKI prediction. All hypotheses were tested at the significance level $\mathrm{a}=0.05$.

\section{Results}

Seventy-two patients were included in the study, $41(56.9 \%)$ patients presented as newly-diagnosed acute heart failure, 31 (43\%) patients were admitted with an acute decompensation of chronic heart failure. The mean age in the whole cohort was $69 \pm 10.3$ years with a gender distribution $46 / 26$ (male/female). Heart failure with a preserved ejection fraction/heart failure with a reduced ejection fraction and heart failure with a mid-range ejection fraction resp. was identified in 33/23/16 patients. The mean value of systolic blood pressure was $140.6 \pm 30 \mathrm{mmHg}$.
Tab. 1. Baseline patient characteristics.

\begin{tabular}{lccc}
\hline Parameter & AKI $+(\mathrm{n}=20)$ & AKI- $(\mathrm{n}=52)$ & $\mathrm{p}$ \\
\hline Age (years) & $75(64-81.5)$ & $64(60-76)$ & 0.01 \\
Males/Females (n) & $10 / 10$ & $36 / 16$ & 0.16 \\
Arterial hypertension n (\%) & $14(70 \%)$ & $44(84.6 \%)$ & 0.49 \\
Diabetes mellitus n (\%) & $9(45 \%)$ & $23(44.2 \%)$ & 0.78 \\
ACS n (\%) & $8(40 \%)$ & $32(61.5 \%)$ & 0.27 \\
De novo HF n (\%) & $10(50 \%)$ & $31(59.6 \%)$ & 0.66 \\
ADCHF n (\%) & $9(45 \%)$ & $22(42.3 \%)$ & 0.68 \\
LVEF<40 \% n (\%) & $11(55 \%)$ & $12(23.1 \%)$ & 0.01 \\
LVEF 40-49 \% n (\%) & $6(30 \%)$ & $10(19.2 \%)$ & 0.35 \\
LVEF $\geq 50 \% \mathrm{n}(\%)$ & $3(15 \%)$ & $30(57.7 \%)$ & 0.001 \\
\hline
\end{tabular}

ACS - acute coronary syndrome, ADCHF - acute decompensated chronic heart failure, HF - heart failure, LVEF - left ventricle ejection fraction. Unless otherwise specified, the data are presented as medians with interquartile range.

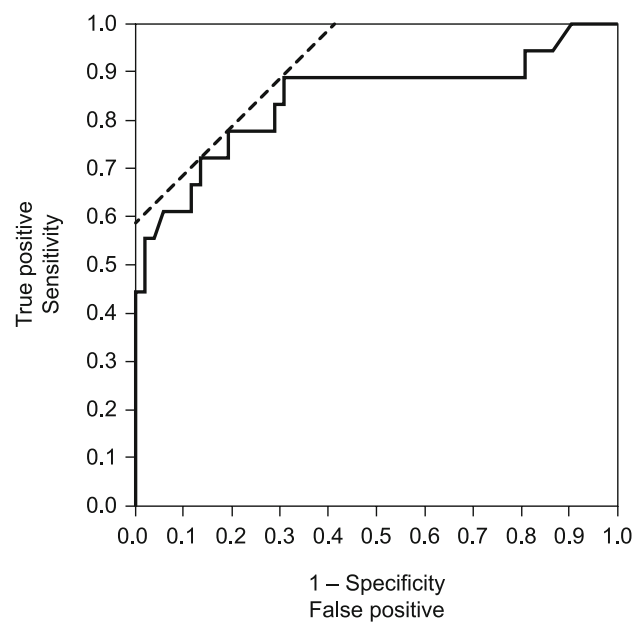

Fig. 1. ROC analysis for AKI prediction in patient with AHF (u-NGAL at admission).

In the $20(27 \%)$ patients, the development of AKI within 72 hours was observed. Patients with AKI+ were older ( 75 vs 64 years (median), $\mathrm{p}=0.01$ ), with lower BMI (28 vs $29.5 \mathrm{~kg} / \mathrm{m}^{2}$ (median), $\mathrm{p}=0.04)$, more often in the HFrEF group $(45.8 \%, \mathrm{p}=0.003)$. The baseline characteristics are shown in the Table 1. Median of $\mathrm{u}-$ NGAL levels at the admission in the AKI+ group was significantly higher than in the AKI- group ( $154 \mathrm{vs} 19.5 \mathrm{ng} / \mathrm{mL}$; $<<0.0001$ ). Median of u-TIMP2 levels was in the AKI+ group 194.1 vs 42.5 $\mathrm{ng} / \mathrm{mL}$ in the AKI-group of patients $(\mathrm{p}<0.0001)$. Median of $\mathrm{u}-\mathrm{IGFBP7}$ levels was in the AKI+ 379 versus $92.4 \mathrm{pg} / \mathrm{mL}$ in the AKI-patients $(\mathrm{p}<0.0001)$ (Tab. 2). The sensitivity for $\mathrm{u}-\mathrm{NGAL}$ at the admission was $72.7 \%$, with a specificity of $80.4 \%$ and area

Tab. 2. Selected laboratory characteristics of patients.

\begin{tabular}{lccc}
\hline Parameter & AKI $+(\mathrm{n}=20)$ & AKI- $(\mathrm{n}=52)$ & $\mathrm{p}$ \\
\hline Haemoglobin $(\mathrm{g} / \mathrm{L})$ & $120(107-132.7)$ & $138.5(127-149)$ & 0.006 \\
Serum creatinine at admission $(\mu \mathrm{mol} / \mathrm{L})$ & $105(90-129.7)$ & $91(75-115)$ & 0.14 \\
Urinary NGAL at admission $(\mathrm{ng} / \mathrm{mL})$ & $152(60-304)$ & $19.5(4-51.2)$ & $<.0001$ \\
Urinary TIMP-2 at admission $(\mathrm{ng} / \mathrm{mL})$ & $194.1(144.6-258)$ & $42.5(26.7-64.4)$ & $<.0001$ \\
Urinary IGFBP-7 at admission $(\mathrm{pg} / \mathrm{mL})$ & $379(254-691)$ & $92.4(68-141.6)$ & $<.0001$ \\
Serum NTproBNP at admission $(\mathrm{pg} / \mathrm{mL})$ & $5883(2332-10405)$ & $2079(443-5609)$ & 0.007 \\
\hline
\end{tabular}

IGFBP - insulin-like factor binding protein, NGAL - neutrophil gelatinase associated lipocalin, NTproBNP - N-terminal fragment of natriuretic peptide B, TIMP - tissue inhibitor of metalloproteinase. Unless otherwise specified, the data are presented as medians with interquartile range. 


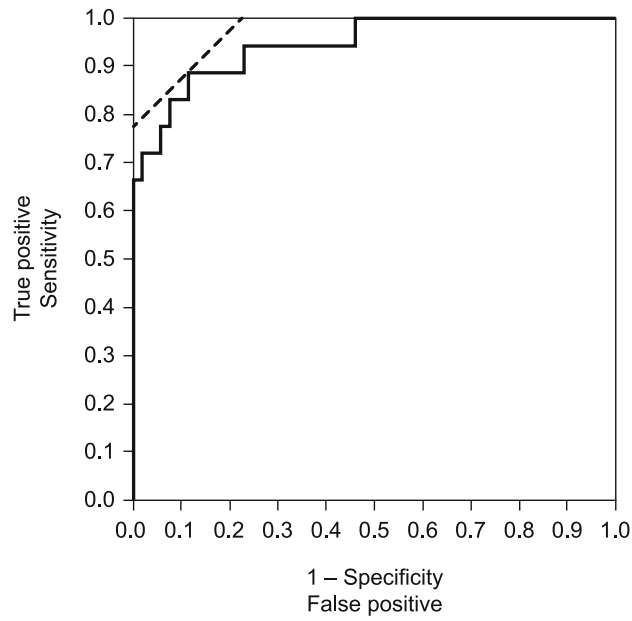

Fig. 2. ROC analysis for AKI prediction in patient with AHF (IGFBP-7 at admission).

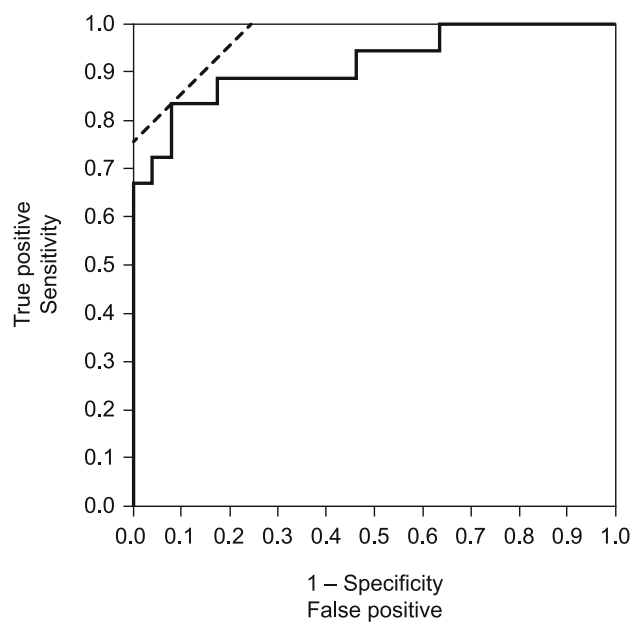

Fig. 3. ROC analysis for AKI prediction in patient with AHF (TIMP2 at admission).

under the ROC curve (AUC) $0.84(\mathrm{p}=0.0003)$. The sensitivity for IGFBP-7 at admission was $88.2 \%$, specificity of $77.4 \%$ and area under the ROC curve 0.94 ( $\mathrm{p}=0.0001)$. The sensitivity for TIMP-2 at the admission was $76.4 \%$ with a specificity of $69.8 \%$, AUC ROC $0.89(\mathrm{p}=0.0003)$ (Figs 1, 2, 3).

Except BMI, parameters such as: age, urinary NGAL, urinary TIMP2, urinary IGFBP7, serum haemoglobin and left ventricular ejection fraction were associated with the AKI development (Tab. $3)$. Hospitalisation death rate was higher in the AKI+ group of the patients. Five in-hospital deaths were recorded, all of them in the AKI+ group $(\mathrm{p}<0.0001)$.

\section{Discussion}

Acute cardiorenal syndrome represents an acute exacerbation of heart failure leading to an acute renal impairment. Renal venous congestion together with an accelerated neurohumoral response (increased activation of the sympathetic nervous system and the renin-
Tab. 3. Selected parameters of logistic regression for AKI in patients.

\begin{tabular}{lccc}
\hline Parameter & OR & $95 \%$ CI & $\mathrm{p}$ \\
\hline u-NGAL & 1.01 & $1.002-1.019$ & 0.01 \\
s-NTproBNP & 1.59 & $1.07-2.11$ & 0.08 \\
u-TIMP-2 & 1.5 & $1.09-1.89$ & 0.009 \\
u-IGFBP-7 & 1.03 & $0.95-1.12$ & 0.04 \\
Age & 2.3 & $1.04-3.2$ & 0.04 \\
LVEF & 1.27 & $0.94-1.6$ & 0.05 \\
s-haemoglobin & 3.7 & $1.04-6.3$ & 0.05 \\
\hline
\end{tabular}

$\mathrm{CI}$ - confidence interval, IGFBP- insulin-like factor binding protein, LVEF - left ventricle ejection fraction NGAL - neutrophil gelatinase associated lipocalin, NTproBNP - N-terminal fragment of natriuretic peptide $\mathrm{B}, \mathrm{OR}$ - odds ratio, TIMP- tissue inhibitor of metalloproteinase

angiotensin-aldosterone axis) are considered the key pathophysiological mechanisms of AKI in AHF (8). Demešová et al documented a significant relationship between the renal congestion due to heart failure and decreased glomerular filtration rate (9). The worsening renal function was reported from $11-40 \%$ patients admitted due to an acute heart failure, and was associated with a higher hospitalisation and long-term mortality rate (10). In current clinical practice, AKI is typically diagnosed by measuring serum creatinine concentrations. Unfortunately, creatinine is an unreliable indicator during acute changes in kidney functions. Serum creatinine concentrations can vary widely with age, gender, muscle mass, muscle metabolism, medications and hydration status. Serum creatinine concentrations may not change until a significant amount of kidney function has already been lost. During acute changes in glomerular filtration, serum creatinine concentration does not accurately depict kidney function until steady state equilibrium has been reached (11). Our study documented, that the increase in serum creatinine levels occurred after 24 hours. By a logistic regression analysis, age, haemoglobin concentration and left ventricular ejection fraction at the admission were identified as clinically commonly available variables for identification the patients at the risk of AKI. We performed ROC analysis of urinary NGAL, TIMP2 and IGFBP7 in the AKI risk assessment. IGFBP-7 was the best predictor of AKI (AUC 0.94).

NGAL is a widely expressed $25-\mathrm{kD}$ protein of the lipocalin family, originally isolated from specific neutrophil granules. It is produced by epithelial cells particularly of distal renal tubule after ischemic and nephrotoxic kidney injury. Elevated NGAL protein is detectable in the urine after 30-120 minutes after injury. The reason for this rapid NGAL production is protective and proliferative effect on kidney epithelium, where binding to iron chelates reduces cell damage (12). We documented, that urinary NGAL at the admission identified patients with development of AKI with sensitivity of $72.7 \%$ and specificity of $80.4 \%$ (AUC ROC 0.84 ).

The most positive results for NGAL worldwide are studies, where the time of inducing impulse is precisely defined. Bennett et al demonstrated plasma and urinary NGAL as an early predictor of renal damage after a cardiac surgery (13). Bachorzewska-Gajewska et al identified NGAL as an early predictor of acute kidney injury after percutaneous coronary interventions (14).

On the other hand, several papers did not confirm superiority of NGAL to serum creatinine in the prediction of clinically relevant worsening renal function and adverse events during hospitalisation $(15,16)$. 
In the multicentre prospective study GALLANT with 186 acute heart failure patients, plasma NGAL at the time of discharge was a strong prognostic indicator of 30 days outcomes and it was superior to the conventional measures of renal function such as: serum creatinine and glomerular filtration rate (17).

The best sensitivity and specificity ratio to estimate the risk of AKI with AUC of 0.94 was found for IGFBP- 7, comparable value for TIMP-2. Both of these biomarkers are potent inducers of cycle cell arrest in the G1 phase, a condition prior to the development of the injury leading to the AKI. In case of inflammation, ischemia, or other insult, renal tubular cells may enter the cycle cell arrest period in G1 phase to prevent cell division, increased cell vulnerability to necrosis or apoptotic death. The kidney cells also repair genome damage (18). It seems that diagnostic accuracy of TIMP-2 and IGFBP-7 in the AKI identification is not adversely affected by comorbidities (19). These biomarkers identified the risk of AKI in patients with acute heart failure and their higher values were associated with a higher mortality and the need for dialysis within 9 months of admission $(20,21,22)$.

\section{Conclusion}

The study showed that acute heart failure patients developing acute kidney injury during indexed hospitalization had higher urinary concentrations of NGAL, TIMP-2, IGFBP-7 already at the admission. The application of the evaluation of novel AKI biomarkers in the decision-making process of heart failure treatment remains uncertain. Whether the ability to predict worsening of renal functions will influence the diagnostic process, therapy or outcome remains questionable. This information could be helpful for increasing awareness in indication of diagnostic procedures or treatment with documented nephrotoxic potential.

\section{Study limitations}

The main limitation of this study is the small sample size and the fact, that the parameters did not reflect the impact on long-term mortality of study group.

\section{References}

1. Mullens W, Damman K, Testani JM et al. Evaluation of kidney function throughout the heart failure trajectory - a position statement from the Heart Failure Association of the European Society of Cardiology. Eur J Heart Fail 2020.

2. Forman De, Butler J, Wang Y et al. Incidence, predictors at admission and impact of worsening renal function among patients hospitalised with heart failure. J Am Coll Cardiol 2004; 43: 61-67.

3. Zannad F, Rossignol, PAF. Cardiorenal Syndrome Revisited. Circulation 2019; 138: 929-944.

4. Kidney Disease: Improving Global Outcomes (KDIGO) Acute Kindey Injury Work Group. KDIGO Clinical Practice Guideline for Acute Kidney Injury. Kidney Int., Suppl 2012; 2: 1/138.

5. Murray PT, Mehta RL, Shaw A et al. Potential use of biomarkers in acute kidney injury: report and summary of recommendations from the 10th Acute Dialysis Quality Initiative consensus conference. Kidney Int 2014; 85: 513-521.
6. Joannidis M, Forni LG, Haase M et al. Use of Cell Cycle Arrest Biomarkers in Conjunction With Classical Markers of Acute Kidney Injury. Crit Care Med 2019; 47: 820-826.

7. Maizel J, Daubin D, Vong LV et al. Urinary TIMP2 and IGFBP7 Identifies High Risk Patients of Short-Term Progression from Mild and Moderate to Severe Acute Kidney Injury during Septic Shock: A Prospective Cohort Study. Dis Markers 2019; 3471215.

8. Di Nicoló P. The dark side of the kidney in cardio-renal syndrome: renal venous hypertension and congestive kidney failure. Heart Fail Rev 2018; 23: 291-302.

9. Demesova E, Goncalvesova E, Slezak P et al. Functional and circulatory renal changes in advanced heart failure. Bratisl Lek Listy 2015; 116: 83-87.

10. Rangaswami J, Bhalla V, Blair J et al. Cardiorenal Syndrome: Classification, Pathophysiology, Diagnosis, and Treatment Strategies A Scientific Statement from the American Heart Association. Circulation 2019; 139: 840-878.

11. van Duijl TT, Ruhaak LR, de Fijter JW et al. Kidney Injury Biomarkers in an Academic Hospital Setting: Where Are We Now? Clin Biochem Rev 2019; 40: 79-97.

12. Mori K, Nakao K. Neutrophil gelatinase associated lipocalin as the real time indicator of kidney damage. Kidney Int 2007; 71: 967-970.

13. Bennett M, Dent CL, Ma $\mathbf{Q}$ et al. Urine NGAL predicts severity of acute kidney injury after cardiac surgery: a prospective study. Clin J Am Soc Nephrol 2008; 3: 665-673.

14. Bachorzewska-Gajewska H, Malzsyko J, Sitniewska E et al. Neutrophil-gelatinase associated lipocalin and renal function after percutaneous coronary interventions. Am J Nephrol 2006; 26: 287-292.

15. Damman K, Valente MAE, van Veldhuisen DJ et al. Plasma Neutrophil gelatinase associated lipocalin and predicting clinically relevant worsening renal function in acute heart failure. Int J Mol Sci 2017; 18: 1470.

16. Murray PT, Wettersten N, van Veldhuisen DJ et al. Utility of Urine Neutrophil Gelatinase-Associated Lipocalin for Worsening Renal Function during Hospitalization for Acute Heart Failure: Primary Findings of the Urine N-gal Acute Kidney Injury N-gal Evaluation of Symptomatic Heart Failure Study (AKINESIS). J Card Fail 2019; 25: 654-665.

17. Maisel AS, Mueller C, Fitzgerald R et al. Prognostic utility of plasma neutropil gelatinase-associated lipocalin in patients with acute heart failure: the NGAL Evaluation Along with B-type Natriuretic Peptide in acutely decompensated heart failure (GALLANT) trial. Eur J Heart Fail 2011; 13: 846-851.

18. Husi H, Human C. Molecular determinants of acute kidney injury. J Inj Violence Res. 2015; 7: 75-86.

19. Heung M, Ortega LM, CHawla LS et al. Common chronic conditions do not affect performance of cycle cell arrest biomarkers for risk stratification of acute kidney injury. Nephrol Dial Transplant 2016; 31: 1633-1640.

20. Schanz M, Shi J, Wasser Ch. et al. Urinary TIMP2xIGFBP7 for risk prediction of acute kidney injury in decompensated heart failure. Clin Cardiol 2017; 40: 485-491.

21. Koyner JL, Shaw AD, Chawla LS et al. Tissue inhibitor metalloproteinase 2 (TIMP2)*IGF-Binding Protein 7 (IGFBP7) levels are associated with adverse longterm outcomes in patients with AKI. J Am Soc Nephrol 2015; 26: 1747-1754.

22. Xie Y, Ankawi G, Yang B et al. Tissue inhibitor metalloproteinase-2 (TIMP-2). IGF-binding protein-7 (IGFBP7) levels are associated with adverse outcomes in patients in the intensive care unit with acute kidney injury. Kidney Int 2019; 95: 1486-1493.

Received February 14, 2019. Accepted February 10, 2020. 